International Journal of Applied Mathematical Research, 1 (4) (2012) 422-432

(C) Science Publishing Corporation

www.sciencepubco.com/index.php/IJAMR

\title{
Hyers-Ulam Stability of Linear and Nonlinear Differential Equations of Second Order
}

\author{
Maher Nazmi Qarawani \\ Department of Mathematics, AlQuds Open University,Palestine, West-Bank \\ Email: mkerawani@qou.edu
}

\begin{abstract}
In this paper we established the Hyers-Ulam stability of a nonlinear differential equation of second order with initial condition. We also proved the Hyers -Ulam stability of a linear differential equation of second order with initial condition.
\end{abstract}

Keywords: Differential equation, Hyers -Ulam Stability, Linear, Nonlinear, Second-order.

\section{Introduction}

In [1], Ulam posed the basic problem of the stability of functional equations: Give conditions in order for a linear mapping near an approximately linear mapping to exist . This problem was partially solved by Hyers in 1941, for approximately additive mappings on Banach spaces [2]. In 1978 Rassias in his work [3], has generalized that result obtained by Hyers.

After then, many mathematicians have extensively investigated the stability problems of functional equations (see $[4,5,6]$ ).

Alsina and Ger [7] were the first mathematicians who investigated the Hyers-Ulam stability of the differential equation $g^{\prime}=g$. They proved that if a differentiable function $y: I \rightarrow R$ satisfies $\left|y^{\prime}-y\right| \leq \varepsilon$ for all $t \in I$, then there exists a differentiable function $g: I \rightarrow R$ satisfying $g^{\prime}(t)=g(t)$ for any $t \in I$ such that $|g-y| \leq 3 \varepsilon$,for all $t \in I$. This result of Alsina and Ger has been generalized by Takahasi et al. [8] to the case of the complex Banach space valued differential equation $y^{\prime}=\lambda y$. 
Furthermore, the results of Hyers-Ulam stability of differential equations of first order were also generalized by Miura et al. [9], Wang et al. [10], and Jung [11]. In the paper [12] Jung proved the Hyers-Ulam stability for Legendre's differential equation $\left(1-x^{2}\right) y^{\prime \prime}-2 x y+p(p+1) y=0$ when the function $y(x)$ has a power series form. In his paper Li [13] has established the HyersUlam stability of the equation $y^{\prime \prime}=\lambda^{2} y$, while Gavruta et al. [14] proved the Hyers-Ulam stability of the equation $y^{\prime \prime}+\beta(x) y=0$ with boundary and initial conditions. Li and Shen [15] proved the stability of the nonhomogeneous linear differential equation of second order $y^{\prime \prime}+p(x) y^{\prime}+q(x) y+r(x)=0$ in the sense of the Hyers and Ulam . In the paper [16] Javadian et al. have proved the Hyers and Ulam stability of the nonhomogeneous linear differential equation of second order $y^{\prime \prime}+p(x) y^{\prime}+q(x) y=f(x)$ in a complex Banach space with the condition that there exists a solution of the corresponding homogeneous equation.

In this paper we investigate the Hyers-Ulam stability of the following nonlinear differential equation of second order

$$
z^{\prime \prime}+p(x) z^{\prime}+q(x) z=h(x)|z|^{\beta} e^{\left(\frac{\beta-1}{2}\right) \int p(x) d x} \operatorname{sgn} z \quad, \beta \in(0,1)
$$

with the initial conditions

$$
z\left(x_{0}\right)=0=z^{\prime}\left(x_{0}\right)
$$

where $q \in C^{0}(I),, h, p \in C^{1}(I), I=\left[x_{0}, x\right] \subseteq \mathbb{R}, x_{0}>0, p(x)>0$, and $h(x)$ is a bounded for all sufficiently large $x$ in $R$. Moreover we proved the Hyers-Ulam stability of the linear differential equation of second order

$$
z^{\prime \prime}+p(x) z^{\prime}+(q(x)-\alpha(x)) z=0
$$

with the initial conditions

$$
z\left(x_{0}\right)=0=z^{\prime}\left(x_{0}\right)
$$

where $\alpha(x)$ is a bounded function for all sufficiently large $x$ in $R$.

It should be note here that we may assume that $z>0$ in equation (1) because if $z<0$ we set $z=-u, u>0$. So we will consider in future the equation

$$
z^{\prime \prime}+p(x) z^{\prime}+q(x) z=h(x) z^{\beta} e^{\left(\frac{\beta-1}{2}\right) \int p(x) d x} \quad, \beta \in(0,1)
$$

\section{Preliminaries and Auxiliary Results}

Definition 2.1: We will say that the equation (3) has the Hyers -Ulam stability with the initial conditions (4) if there exists a positive constant $K>0$ with the following property: 
For every $\varepsilon>0, z \in C^{2}(I)$ where $x$ is sufficiently large in $\mathbb{R}$, if

$$
\left|z^{\prime \prime}+p(x) z^{\prime}+(q(x)-\alpha(x)) z\right| \leq \varepsilon
$$

then there exists some solution $w \in C^{2}(I)$ of the equation (5), such that $|z(x)-w(x)| \leq K \varepsilon$ and satisfies the initial conditions

$$
w\left(x_{0}\right)=0=w^{\prime}\left(x_{0}\right)
$$

Definition 2.2: We say that equation (5) has the Hyers -Ulam stability with initial conditions (4) if there exists a positive constant $K>0$ with the following property:

For every $\varepsilon>0, z \in C^{2}(I)$ where $x$ is sufficiently large in $\mathbb{R}$, if

$$
\left|z^{\prime \prime}+p(x) z^{\prime}+q(x) z-h(x) z^{\beta} e^{\left(\frac{\beta-1}{2}\right) \int p(x) d x}\right| \leq \varepsilon
$$

then there exists some solution $w \in C^{2}(I)$ of the equation (5) and

$$
w\left(x_{0}\right)=w^{\prime}\left(x_{0}\right)=0
$$

such that $|z(x)-w(x)| \leq K \varepsilon$.

Definition 2.3: We will say that the equations (3),(5) have the Hyers -Ulam asymptotic stability with the initial conditions (4) if the equation is stable in the sense of Hyers and Ulam and $\lim _{x \rightarrow \infty}(z(x)-w(x))=0$.

The author in his work [17] has proved the following Lemma and Theorem.

Lemma 2.1: (see [17]) A substitution $z(x)=y(x) \exp \left(-\frac{1}{2} \int p(x) d x\right)$ reduces the equations (3) and (5) to the equations (10) and (11), respectively

$$
\begin{gathered}
y^{\prime \prime}+y=\alpha(x) y \\
y^{\prime \prime}+y=h(x) y^{\beta} \quad, \beta \in(-1,1) \backslash\{0\}
\end{gathered}
$$

where

$$
q(x)-\frac{1}{4} p^{2}(x)-\frac{1}{2} p^{\prime}(x)=1
$$

Theorem 2.1(see [17]) Suppose that $h(x)$ is a continuously differentiable function, bounded for all sufficiently large $x \in R$, and that the integral $\int_{x_{0}}^{\infty}\left|h^{\prime}(x)\right| d x$ is convergent then any solution of the equation (11) is bounded as $x \rightarrow \infty$. 
Proof. Multiplying both sides of the equation (11) by $y^{\prime}$ and integrate the result we get

$$
\begin{array}{r}
y^{\prime 2}(x)+y^{\prime 2}(x)=y^{\prime 2}\left(x_{0}\right)+y^{2}\left(x_{0}\right)-\frac{2 h\left(x_{0}\right) y^{\beta+1}\left(x_{0}\right)}{\beta+1} \\
+\frac{2 h(x) y^{\beta+1}(x)}{\beta+1}-\frac{2}{\beta+1} \int_{x_{0}}^{x} h^{\prime}(t) \cdot y^{\beta+1}(t) d t
\end{array}
$$

Hence

$$
y^{2}(x) \leq y^{\prime 2}(x)+y^{2}(x) \leq A_{x_{0}}+\frac{2|h(x)||y(x)|^{\beta+1}}{\beta+1}+\frac{2}{\beta+1} \int_{x_{0}}^{x}\left|h^{\prime}(t)\right| \cdot|y(t)|^{\beta+1} d t
$$

where $A_{x_{0}} \geq 0$ is an expression dependent only on $x_{0}$.

Let $M=\max _{x_{0} \leq t \leq x}|y(t)|$, and without loss of generality we may assume that $M \geq a_{0}>0$, otherwise the theorem is proved. Since $h(x)$ is bounded we get

$$
\begin{aligned}
M^{1-\beta} \leq \frac{A_{x_{0}}}{M^{\beta+1}}+\frac{2 B_{0}}{\beta+1}+\frac{2}{\beta+1} \int_{x_{0}}^{x}\left|h^{\prime}(t)\right| d t \leq & \frac{A_{x_{0}}}{a_{0}}+\frac{2 B_{0}}{\beta+1} \\
& +\frac{2}{\beta+1} \int_{x_{0}}^{\infty}\left|h^{\prime}(t)\right| d t
\end{aligned}
$$

Since the integral $\int_{x_{0}}^{\infty}\left|h^{\prime}(x)\right| d x$ converges, we obtain

$$
|y(x)| \leq M \leq C^{\frac{1}{1-\beta}}, \beta \in(-1,1) \backslash\{0\}
$$

Therefore $y(x)$ is bounded for $x \rightarrow \infty$.

In the following theorem the author has established sufficient conditions for boundedness of the solutions of the equation (10) which are similar to those obtained in [18].

Theorem 2.2 Suppose that $|\alpha(x)| \leq L$ for all $x \geq x_{0}$. If $L<1$ then any solution of the equation (10) is bounded as $x \rightarrow \infty$.

Proof. Multiplying both sides of the equation (10) by $y^{\prime}$ and integrating the result, we obtain

$$
\int_{x_{0}}^{x} y^{\prime}(t) \cdot y^{\prime \prime}(t) d t+\int_{x_{0}}^{x} y(t) \cdot y^{\prime}(t) d t=2 \int_{x_{0}}^{x} \alpha(t) \cdot y(t) y^{\prime}(t) d t
$$


Since $\alpha(x)$ is bounded we get

$$
\begin{aligned}
y^{2}(x) & \leq y^{\prime 2}(x)+y^{2}(x) \leq A_{x_{0}}+2 \int_{x_{0}}^{x} \alpha(t) \cdot y(t) y^{\prime}(t) d t \\
& \leq A_{x_{0}}+L y^{2}(x)
\end{aligned}
$$

It follows that

$$
y^{2} \leq \frac{A_{x_{0}}}{(1-L)}
$$

Therefore $y(x)$ is bounded for $x \rightarrow \infty$.

\section{Main Results on Hyers-Ulam stability}

Theorem 3.1 Suppose $|\alpha(x)| \leq L<1$ for all $x \geq x_{0}$, and that $y \in C^{2}(I)$, such that satisfies the inequality

$$
\left|y^{\prime \prime}+y-\alpha(x) y\right| \leq \varepsilon
$$

with the initial condition

$$
y\left(x_{0}\right)=0=y^{\prime}\left(x_{0}\right)
$$

Then the equation (10) has the Hyers-Ulam stability with initial condition (14).

Proof. suppose that $\varepsilon>0$ and $y \in C^{2}(I)$ satisfies the inequation (13) with the initial conditions (14) and $M=\max _{x \geq x_{0}}|y(x)|$.

We will show that there exists a function $w(x) \in c^{2}(I)$ satisfying the equation (10) and the initial condition (7) such that $|z(x)-w(x)| \leq k \varepsilon$.

From the inequality (13) we have

$$
-\varepsilon \leq y^{\prime \prime}+y-\alpha(x) y \leq \varepsilon
$$

Multiply the inequality (15) by $y^{\prime}$ and then integrate we obtain

$$
-2 \varepsilon y \leq y^{\prime 2}(x)+y^{2}(x)-2 \int_{x_{0}}^{x} \alpha(t) y y^{\prime} d t \leq 2 \varepsilon y
$$

From which we get that

$$
\begin{aligned}
y^{2}(x) & \leq 2 \varepsilon y+2 \int_{x_{0}}^{x} \alpha(t) y y^{\prime} d t=2 \varepsilon y+\alpha\left(x^{*}\right) y^{2} \leq 2 \varepsilon y+\alpha\left(x^{*}\right) y^{2} \\
& \leq 2 \varepsilon M+L M^{2}
\end{aligned}
$$


Therefore

$$
M \leq \frac{2 \varepsilon}{1-L}
$$

Hence $|y(x)| \leq k \varepsilon$, for all $x \geq x_{0}$. Obviously , $w_{0}(x)=0$ satisfies the equation (10) and the zero initial condition (14) such that

$$
\left|y(x)-w_{0}(x)\right| \leq k \varepsilon
$$

Hence the equation (10) has the Hyers-Ulam stability with initial condition (14).

Corollary 3.1: Suppose $|\alpha(x)| \leq L<1$ for all $x \geq x_{0}, z \in C^{2}(I)$ and satisfies the inequality (6) with the initial condition (4). If the integral $\int_{x_{0}}^{\infty} p(x) d x \quad$ converges then the equation (3) has the Hyers-Ulam stability with initial condition (4).

Proof. Suppose that $z \in C^{2}(I)$ satisfies the inequality

$$
\left|z^{\prime \prime}+p(x) z^{\prime}+(q(x)-\alpha(x)) z\right| \leq \varepsilon
$$

From the Theorem 3.1 it follows that the equation (10) has the Hyers-Ulam stability with initial condition (14) and according to the substitution in Lemma 2.1 it follows that the equation (3) has the Hyers-Ulam stability with initial condition (4).

Corollary 3.2 Suppose $|\alpha(x)| \leq L<1$ for all $x \geq x_{0}, z \in C^{2}(I)$ and satisfies the inequality (6) with the initial condition (4) and $\int_{x_{0}}^{\infty} p(x) d x=\infty$ , then the equation (3) has the Hyers-Ulam asymptotic stability with initial condition (4).

Proof. From the Corollary 3.1 it follows that the equation (3) has the Hyers-Ulam stability with initial condition (4). Since $\int_{x_{0}}^{\infty} p(x) d x=\infty$ then according to the substitution in Lemma 2.1 it follows that the equation (3) has the Hyers-Ulam asymptotic stability with initial condition (4).

Theorem 3.2 Suppose $|h(x)| \leq A$ for all $x \geq x_{0}$, and that $y \in C^{2}(I)$, such that satisfies the inequality

$$
\left|y^{\prime \prime}+y-h(x) y^{\beta}\right| \leq \varepsilon \quad, \beta \in(0,1)
$$

with the initial condition

$$
y\left(x_{0}\right)=0=y^{\prime}\left(x_{0}\right)
$$

If $A<\frac{(\beta+1)}{2}\left(\max _{x \geq x_{0}}|y(x)|\right)^{-\beta}$,for $x \geq x_{0}$, then the equation

$$
y^{\prime \prime}+y=h(x) y^{\beta} \quad, \beta \in(0,1)
$$

has the Hyers-Ulam stability with initial condition (17). 
Proof. suppose that $\varepsilon>0, y \in C^{2}(I)$ satisfies the inequation (16) with the initial conditions (17) and that $M=\max _{x \geq x_{0}}|y(x)|$.

We will show that there exists a function $w(x) \in c^{2}(I)$ satisfying the equation (18) and the initial condition (17) such that $|z(x)-w(x)| \leq k \varepsilon$.

From the inequality (16) we have

$$
-\varepsilon \leq y^{\prime \prime}+y-h(x) y^{\beta} \leq \varepsilon
$$

Multiply the inequality (19) by $y^{\prime}$ and then integrate we obtain

$$
-2 \varepsilon y \leq y^{\prime 2}(x)+y^{2}(x)-2 \int_{x_{0}}^{x} h(x) y^{\beta} y^{\prime} d t \leq 2 \varepsilon y
$$

From which we get that

$$
y^{2}(x) \leq 2 \varepsilon y+2 \int_{x_{0}}^{x} h(t) y^{\beta} y^{\prime} d t=2 \varepsilon y+\frac{2 h\left(x^{*}\right) y^{\beta+1}}{\beta+1} \leq 2 \varepsilon M+\frac{2 A M^{\beta+1}}{\beta+1}
$$

Therefore

$$
M \leq \frac{2 \varepsilon}{1-\frac{2 A M^{\beta}}{\beta+1}}
$$

Hence $|y(x)| \leq k \varepsilon$, for all $x \geq x_{0}$. Obviously , $w_{0}(x)=0$ satisfies the equation (18) and the zero initial condition (17) such that

$$
\left|y(x)-w_{0}(x)\right| \leq k \varepsilon
$$

Thus the equation (18) has the Hyers-Ulam stability with initial condition (17).

Corollary 3.3 Assume that $h(x)$ and $z(x)$ satisfy the conditions of Theorem 3.2, and the inequality (8) with the initial condition (2).

If $A<\frac{(\beta+1)}{2}\left(\max _{x \geq x_{0}}|y(x)|\right)^{-\beta}$,for $x \geq x_{0}$ and the integral $\int_{x_{0}}^{\infty} p(x) d x$ converges then the equation (5) has the Hyers-Ulam stability with initial condition (2). Moreover, if the integral $\int_{x_{0}}^{\infty} p(x) d x=\infty$ then the equation (5) has the Hyers-Ulam asymptotic stability with initial condition (2).

Proof. Suppose that $z \in C^{2}(I)$ satisfies the inequality (8) with the initial condition (2).

Then from the Theorem 3.2 it follows that the equation (18) has the HyersUlam stability with initial condition (17), and according to the substitution used in Lemma 2.1 it follows that the equation (5) has the Hyers-Ulam stability with initial condition (2). Now if $\int_{x_{0}}^{\infty} p(x) d x=\infty$, then the equation (5) has the Hyers-Ulam asymptotic stability with initial condition (2). 
Now we illustrate the Theorem by the following example.

Example 3.1 Consider the equation

$$
z^{\prime \prime}+\frac{2}{x} z^{\prime}+z=\frac{e^{-x / 2} z^{1 / 2}}{\sqrt{x}}
$$

with the initial condition

$$
z\left(x_{0}\right)=0=z^{\prime}\left(x_{0}\right)
$$

If we set $z(x)=\frac{y(x)}{x}$ in the the equation (20) we obtain

$$
y^{\prime \prime}(x)+y(x)=e^{-x / 2} y^{1 l 2}
$$

We let $y(x)=\left(x-x_{0}\right)^{2} e^{-x}$ and estimate the difference

$$
\left|y^{\prime \prime}(x)+y(x)-e^{-x / 2} y^{1 l 2}\right|=\left|\frac{2-5\left(x-x_{0}\right)+2\left(x-x_{0}\right)^{2}}{e^{x}}\right| \leq \varepsilon
$$

Now we may choose the number $x_{0}$ sufficiently large such that the inequality (23) will satisfy for any $x \geq x_{0}$ and for any $\varepsilon>0$.

Hence $y(x)=\left(x-x_{0}\right)^{2} e^{-x}$ is an approximate solution of the equation (20) satisfying the zero initial condition

$$
y\left(x_{0}\right)=0=y^{\prime}\left(x_{0}\right)
$$

Now we have

$$
h(x)=e^{-x / 2} \leq 1<\frac{3 e}{8}<\frac{3}{4}\left(\max _{x \geq x_{0}}|y(x)|\right)^{-\frac{1}{2}}=\frac{3 e^{1+\frac{x_{0}}{2}}}{8} .
$$

Therefore

$$
M \leq k \varepsilon \quad, \text { where } \frac{6 e^{\left(1+\frac{x_{0}}{2}\right)}}{3 e^{\left(1+\frac{x_{0}}{2}\right)}-8}>0
$$

It is clear that $z_{0} \equiv 0$ satisfies the zero initial condition and the inequality $\left|y(x)-z_{0}(x)\right| \leq k \varepsilon$. Thus the equation (20) has the Hyers-Ulam stability. Moreover, since $\lim _{x \rightarrow \infty}\left|y(x)-z_{0}(x)\right|=0$, then it also is asymptotically stable in the sense of Hyers and Ulam as $\mathrm{x} \rightarrow \infty$. Now since the integral $\int_{1}^{\infty} p(x) d x=$ $\int_{1}^{\infty} \frac{2}{x} d x=\infty$, then by Lemma it follows that the equation (20) has the HyersUlam stability with zero initial condition (21). Moreover the equation (20) is asymptotically stable in the sense of Hyers and Ulam as $x \rightarrow \infty$. 


\section{Special Case of the equation (5)}

Now consider a special case of the equation (5)

$$
x^{2} z^{\prime \prime}+2 \lambda x z^{\prime}+\left[x^{2}+\lambda(\lambda-1)\right] z=h(x) x^{2+\lambda(\beta-1)} z^{\beta}
$$

where $\lambda>0, \quad \beta \in(0,1)$, and it satisfies the initial condition

$$
z\left(x_{0}\right)=0=z^{\prime}\left(x_{0}\right)
$$

It should be note that the equation (25) is a special case of the equation (5) with $p(x)=\frac{2 \lambda}{x}$ and $q(x)=\frac{x^{2}+\lambda(\lambda-1)}{x^{2}}$. So if we let $z(x)=\frac{y(x)}{x^{\lambda}} \quad, \lambda \succ 0$, then the equation (25) is reduced to the equation (18) with $y\left(x_{0}\right)=0=y^{\prime}\left(x_{0}\right)$.

Theorem 4.1 Suppose that the conditions of the Theorem 3.2 hold, the integral $\int_{x_{0}}^{\infty} p(x) d x$ converges and that $z \in C^{2}(I)$ and satisfies the inequality

$$
\left|x^{2} z^{\prime \prime}+2 \lambda x z^{\prime}+\left[x^{2}+\lambda(\lambda-1)\right] z-h(x) x^{2+\lambda(\beta-1)} z^{\beta}\right| \leq \varepsilon
$$

then the equation (25) has the Hyers-Ulam stability with initial condition (26). Moreover, if the integral $\int_{x_{0}}^{\infty} p(x) d x=\infty$ then the equation (25) has the Hyers-Ulam asymptotic stability with the initial condition (26).

Proof. It follows from the Theorem 3.2 and Corollary 3.3

Example 4.1 Consider the equation

$$
x^{2} z^{\prime \prime}+x z^{\prime}+\left(x^{2}-\frac{1}{4}\right) z=x^{7 / 4} e^{-x / 2} z^{1 / 2}
$$

with the initial condition

$$
z\left(x_{0}\right)=0=z^{\prime}\left(x_{0}\right)
$$

Setting $z(x)=\frac{y(x)}{\sqrt{x}}$ in the equation (27) we get

$$
y^{\prime \prime}(x)+y(x)=e^{-x / 2} y^{1 l 2}
$$

If we apply the same argument used in Example 3.1 for the function $y(x)=$ $\left(x-x_{0}\right)^{2} e^{-x} \quad$ we can show that it satisfies the inequality

$$
\left|y^{\prime \prime}(x)+y(x)-e^{-x / 2} y^{1 l 2}\right|<\varepsilon
$$

with initial condition $y\left(x_{0}\right)=0=y^{\prime}\left(x_{0}\right)$, and the inequality

$$
M \leq k \varepsilon \quad, \text { where } k=\frac{6 e^{\left(1+\frac{x_{0}}{2}\right)}}{3 e^{\left(1+\frac{x_{0}}{2}\right)}-8}>0
$$

Therefore, we get the Hyers-Ulam stability and asymptotic stability for the equation (27). 


\section{Conclusion}

In this paper we obtained sufficient criteria for Hyers-Ulam stability of linear and nonlinear differential equations of second Order with zero initial conditions.

\section{ACKNOWLEDGEMENTS}

The author is deeply grateful to the editor professor Belal Batiha for useful comments.

\section{References}

[1] S.-M. Ulam, Problems in Modern Mathematics , Science Edition, John Wiley \& Sons, New York, USA, 1964.

[2] D. H . Hyers, On the stability of the linear functional equation, Proceedings of the National Academy of Sciences of the United States of America, 27 (1941) 222-224.

[3] T. M . Rassias, On the stability of the linear mapping in Banach spaces, Proceedings of the American Mathematical Society, 72 (2) (1978) 297300.

[4] T. Miura, S.-E. Takahasi, H. Choda, On the Hyers-Ulam stability of real continuous function valued differentiable map, Tokyo J. Math. 24 (2001) $467-476$.

[5] S. M. Jung, On the Hyers-Ulam-Rassias stability of approximately additive- mappings, J. Math. Anal. Appl. 204 (1996) 221-226.

[6] C. G. Park, On the stability of the linear mapping in Banach modules , J. Math. Anal. Appl. 275 (2002) 711-720.

[7] C. Alsina, R. Ger, On some inequalities and stability results related to the exponential function, J. Inequal Appl. 2 (4) (1998) 373-380.

[8] E. Takahasi , T. Miura, S. Miyajima, On the Hyers-Ulam stability of the Banach space-valued differential equation $y^{\prime}=\lambda y$, Bulletin of the Korean Mathematical Society 39(2002) 309-315.

[9] T. Miura, S. Miyajima, S.-E. Takahasi, A characterization of Hyers-Ulam stability of first order linear differential operators, J. Math. Anal. Appl. 286 (2003) 136-146. 
[10] G. Wang, M. Zhou, L. Sun, Hyers-Ulam stability of linear differential equations of first order, Appl. Math. Lett. 21(2008) 1024-1028.

[11] S.-M. Jung, Hyers-Ulam stability of linear differential equations of first order, J. Math. Anal. Appl. 311(2005) 139-146.

[12] S.-M. Jung, Legendre's differential equation and Its Hyers-Ulam stability, Abstract and Applied Analysis (2007) doi:10.1155/2007/56419.

[13] Y. Li, Hyers-Ulam Stability of Linear Differential Equations, Thai Journal of Mathematics 8 (2) (2010) 215-219.

[14] P. Gavruta, S. Jung, Y. Li, Hyers-Ulam Stability For SecondOrder Linear Differential Equations With Boundary Conditions, EJDE http://ejde.math.txstate.edu/Volumes/2011/80/gavruta.pdf

[15] Y. Li and Y. Shen, Hyers-Ulam Stability of Nonhomogeneous Linear Differential Equations of Second Order, Int. J. Math. Math. Sci. (2009) doi:10.1155/2009/576852.

[16] A. Javadian, E. Sorouri, G. Kim, M. E. Gordji, Generalized Hyers-Ulam stability of the second-order linear differential equations, J of Applied Mathematics (2011) doi:10.1155/2011/813137.

[17] M. N. Qarawani, Boundedness and asymptotic behaviour of solutions of a second order nonlinear differential equation, J. of Mathematics Research $4(3)$ (2012) 121-128.

[18] A. Bucur, About asymptotic behaviour of solutions of differential equations as $x \rightarrow \infty$, General Mathematics 14 (2) (2006) 55-58. 